\title{
Effects of antioxidants on the microleakage of composite resin restorations after external tooth bleaching
}

\author{
Yanyan $\mathrm{Han}^{1}$, Sisu $\mathrm{Mo}^{1}$, Long Jiang ${ }^{1}$, Yaqin $\mathrm{Zhu}^{1}$
}

Correspondence: Dr. Yaqin Zhu

Email: zyq1590@163.com

\begin{abstract}
'Department of General Dentistry, Ninth People's Hospital, Shanghai Jiao Tong University School of Medicine, Shanghai Key Laboratory of Stomatology, Shanghai, China
\end{abstract}

\begin{abstract}
Objective: To compare the effects of three antioxidants (sodium ascorbate, sodium ascorbate combined with a surfactant, and catalase) on the microleakage of composite resin restorations after external tooth bleaching with $10 \%$ carbamide peroxide. Materials and Methods: Buccal cavities were prepared on the surface of 60 intact premolars, which were randomly divided into six groups. All cavities were filled with composite resin. In group 1, teeth were not bleached; in group 2, cavities were filled immediately after bleaching; in group 3, cavities were delay-filled for 3 weeks; in group 4, cavities were treated with sodium ascorbate after bleaching and then filled; in group 5, cavities were treated with sodium ascorbate combined with surfactant after bleaching and then filled; and in group 6, cavities were treated with catalase after bleaching and then filled. Microleakage of the restorations was measured from sections using a stereomicroscope. Results: Group 1 displayed the least amount of microleakage, while group 2 showed the greatest amount of microleakage $(P<0.05)$. Groups 3 and 4 were similar to group $2(P>0.05)$. However, groups 5 and 6 showed a significantly lower amount of microleakage compared to group $2(P<0.05)$. Conclusion: Microleakage increased significantly after external bleaching with $10 \%$ carbamide peroxide, and decreased when the bleached teeth were treated with sodium ascorbate combined with Tween ${ }^{\circledR} 80$, or with catalase. Catalase was more effective in decreasing microleakage, while delayed filling or treatment with sodium ascorbate alone did not effectively decrease the microleakage.
\end{abstract}

Key words: Antioxidant, composite resin, external tooth bleaching, microleakage

\section{INTRODUCTION}

External tooth bleaching is a conservative and well-accepted technique ${ }^{[1]}$ for whitening teeth with surface and intrinsic staining, which is opening up new avenues and becoming much more prevalent in esthetic dentistry. The idea is that using this technique can effectively make stained teeth whiter through direct application of bleaching agents. Bleaching agents contain peroxide, which bleaches the enamel to change the color of the teeth. To date, various bleaching agents, in different concentrations, have been used in dental clinics to treat tooth discoloration; however, the most commonly used one is $10 \%$ carbamide peroxide due to its superior characteristics as an external bleaching agent, i.e. it is effective, works quickly, and has relatively few side effects while achieving desirable results when used to treat tooth discoloration. ${ }^{[2]}$ Therefore, $10 \%$ carbamide peroxide was chosen as the bleaching agent in this study. However, 10\% carbamide peroxide does have some controversial side effects. Numerous studies have shown that the marginal sealing of composite resin restorations decreased whether the fillings were done before or after bleaching using 10\% carbamide peroxide. ${ }^{[3-5]}$ Also, bleaching may harm the dental pulp, resulting in pulpal reactions such as increased tooth hypersensitivity. ${ }^{[6]}$ As we know, the active

\footnotetext{
How to cite this article: Han Y, Mo S, Jiang L, Zhu Y. Effects of antioxidants on the microleakage of composite resin restorations after external tooth bleaching. Eur J Dent 2014;8:147-53.

Copyright (C) 2014 Dental Investigations Society. DOI: $10.4103 / 1305-7456.130581$
} 
mechanism of bleaching agents mainly depends on a complex oxidation reaction, which releases oxygen and other free radicals. The oxygen and free radicals establish their primary mechanism of action in tooth bleaching by penetrating through the porosities of the enamel prism to the dentin. ${ }^{[7]}$ Increased microleakage of composite resin restorations placed after bleaching may be due to the presence of residual peroxide and residual oxygen that inhibits resin polymerization ${ }^{[8]}$ by interfering with the adhesion of restorative materials.

Several methods have been proposed to decrease microleakage due to bleaching, including removal of the superficial enamel layer, pretreatment of the bleached enamel with alcohol, use of adhesives containing organic solvents, cleansing of the cavity with antioxidants, and a post-bleaching period ranging from $24 \mathrm{~h}$ to 3 weeks. ${ }^{[6]}$ It has been shown that removal of the superficial enamel layer is ineffective because bonding weakens both superficially and internally. ${ }^{[6]}$ One method that is recommended clinically is to delay bonding until 2-3 weeks after bleaching. ${ }^{[9,10]}$ Studies have shown that residual peroxide and oxygen slowly dissipate with time, eventually eliminating any reduction in composite resin bond strength associated with the bleaching, but this is time consuming for practitioners and patients.

Severalantioxidantagents have beenintroduced, suchas sodium ascorbate, ascorbic acid, butylhydroxyanisole, catalase, ethanol, acetone, glutathione, peroxide, $\alpha$-tocopherol, and sodium bicarbonate, in order to better control restoration microleakage; $;^{[11-14]}$ however, only a few of them were found to be effective. We hypothesized that treating bleached enamel with an antioxidant before bonding might reverse the increase in microleakage and might be a viable alternative to delayed restoration after bleaching. Recently, a biocompatible and neutral antioxidant, $10 \%$ sodium ascorbate, was shown to be able to remove the residual peroxide and oxygen, so that compromised bonding to bleached tooth structures, such as dentin or enamel, could be reversed. ${ }^{[15-17]}$ Furthermore, Moosavi et al. ${ }^{[5]}$ demonstrated that the addition of surfactant $(0.2 \%$ Tween ${ }^{\circledR} 80$ ) to a sodium ascorbate formulation could significantly reduce the microleakage after nonvital bleaching. To our knowledge, only one study ${ }^{[18]}$ has investigated the use of catalase in improving the composite-resin bond strength after tooth bleaching. In another study, ${ }^{[19]}$ it was reported that catalase could be used as an adjunct to effectively eliminate residual hydrogen peroxide from the pulp chamber and the surrounding periodontal tissues following intracoronal bleaching of nonvital teeth. In the present study, we examine the effect of catalase on improving adhesion between composite resin and externally bleached teeth and on reduction of microleakage after external tooth bleaching.

The aim of this study is to investigate the effects of three antioxidants (sodium ascorbate, sodium ascorbate combined with a surfactant, and catalase) on the microleakage of composite resin restorations after external tooth bleaching with $10 \%$ carbamide peroxide.

\section{MATERIALS AND METHODS}

\section{Sample collection}

The Ethics Committee of Ninth People's Hospital, Medical College, Shanghai Jiao Tong University, China approved this study. Informed consent was obtained from all the study participants. Sixty intact premolars that had been freshly extracted from healthy orthodontic patients were used. Teeth with caries, hypoplastic areas, or cracks and teeth that had a history of treatment were excluded. After extraction, the soft tissues, dental calculus, and stains were immediately removed from selected surfaces of the teeth. Teeth were then stored in normal saline at $4^{\circ} \mathrm{C}$ for further use. The saline containing the selected teeth was changed every week for 1 month in order to avoid bacterial growth.

\section{Experimental groups}

The stored teeth were randomly divided into five experimental groups ( $n=10$ per group) after bleaching with $10 \%$ carbamide peroxide. An unbleached group $(n=10)$ was used as the control group. Prior to bleaching, tooth surfaces were polished with oil- and fluoride-free fine pumice and water using a brush and a slow-speed hand-piece. All tooth surfaces were then rinsed and dried with an air syringe.

\section{Group 1 (Unbleached or control group)}

Group 1 teeth underwent cavity preparation and restoration filling and were not bleached. Unbleached teeth were immersed in artificial saliva, which was composed of $1 \mathrm{~g}$ sodium carboxymethylcellulose, $4.3 \mathrm{~g}$ xylitol, 0.1 g potassium chloride, $0.1 \mathrm{~g}$ sodium chloride, $0.02 \mathrm{mg}$ sodium fluoride, $5 \mathrm{mg}$ calcium chloride, $5 \mathrm{mg}$ magnesium chloride, $5 \mathrm{mg}$ calcium chloride, $40 \mathrm{mg}$ potassium phosphate, $1 \mathrm{mg}$ potassium thiocyanate, and $100 \mathrm{~g}$ distilled deionized water, ${ }^{[20]}$ at $37^{\circ} \mathrm{C}$ for 10 days. Artificial saliva was changed daily. Box-shaped class $\mathrm{V}$ cavities were made on the buccal surface, around the cementoenamel junction, using a round diamond bur in 
a high-speed hand-piece under air and water-cooling. Occlusal margins and gingival margins of the prepared cavities were located in the enamel and the root, respectively. The prepared cavities were approximately $2 \mathrm{~mm}$ in height, $3 \mathrm{~mm}$ in the mesio-distal direction, and $2 \mathrm{~mm}$ in depth. Adper Easy One self-etching bonding agent (3M/ESPE, St Paul, MN, USA) was used for bonding of the composite resin, according to the manufacturer's instructions. This was spread gently with air for $5 \mathrm{~s}$ and then cured for $10 \mathrm{~s}$ using a pen-style, high-power LED curing light (DENTSPLY, Milford, DE, USA) with an intensity exceeding 950 $\mathrm{mW} \mathrm{cm} \cdot{ }^{[2]}$ Following this, composite resin (Filtek Z350, shade A3, 3M/ESPE) was placed into cavities and polymerized in 1.5-2-mm-thick layers for $20 \mathrm{~s}$. The composite resin was then finished by bur, and the teeth were preserved at $37^{\circ} \mathrm{C}$ in normal saline that was changed daily. The same operator carried out all standard procedures.

\section{Group 2 (Immediate restoration group)}

In group 2 , the teeth were first bleached by the following method and then immediately filled with composite resin. The bleaching procedure is briefly described as follows. The teeth were placed with the occlusal edges positioned downward in a plastic holder containing 10\% carbamide peroxide (Opalescence; Ultradent Products, South Jordan, UT, USA) for $8 \mathrm{~h}$ a day for 10 days. An effective bleaching component of $10 \%$ carbamide peroxide is $3 \%$ hydrogen peroxide. After completion of each daily bleaching procedure, the teeth were rinsed with flowing water and then washed using a soft toothbrush in order to remove the remaining bleaching gel. The bleached teeth were stored in fresh artificial saliva at $37^{\circ} \mathrm{C}$ between treatments. After the final bleaching, washing, and overnight storage in saliva, the cavities were immediately prepared and restored in the bleached teeth as described above for group 1 .

\section{Group 3 (Delayed filling group)}

In group 3, the teeth were bleached as described above and then, after the final washing, immersed in artificial saliva at $37^{\circ} \mathrm{C}$. After 3 weeks, cavities were prepared and composite resin restorations were placed as in group 1.

\section{Group 4 (Sodium ascorbate treated group)}

In group 4 , the teeth were bleached and the cavity prepared immediately as described above for group 2 . Cavities were then treated with $10 \%$ sodium ascorbate using a cotton pellet prior to filling with composite resin. Briefly, $10 \%$ sodium ascorbate was smeared on the bonding surface for $10 \mathrm{~s}$ using a cotton pellet that was then placed into the cavity for $1 \mathrm{~min}$. After removal of the cotton pellet, the cavity was rinsed with distilled water for $2 \mathrm{~min}$ and dried gently with air to prepare it for composite resin restoration.

Group 5 (Sodium ascorbate combined with surfactant treated group)

After bleaching, a novel antioxidant agent composed of $10 \%$ sodium ascorbate and $0.2 \%$ Tween 80 was applied to cavities in the bleached teeth, as described above for group 4 . The samples were restored as described in group 1.

\section{Group 6 (Catalase-treated group)}

The cotton pellet was soaked with $10 \mathrm{mg} / \mathrm{ml}$ catalase. The remaining procedures were the same as those performed in group 4.

\section{Microleakage test}

After restoration, all teeth were immersed in distilled water at $37^{\circ} \mathrm{C}$ for $24 \mathrm{~h}$ and then thermocycled from $5^{\circ} \mathrm{C}$ to $55^{\circ} \mathrm{C}$ for 2000 cycles using a $45 \mathrm{~s}$ dwell time at each temperature. After thermocycling, the specimens were laid aside and dried at room temperature for $24 \mathrm{~h}$. All surfaces of the teeth, except for a 1-mm zone surrounding the restorations' margins, were covered with three coats of nail polish and modeling wax to provide an impermeable barrier to the test fluid (dye). Each layer of the nail polish was $1 \mathrm{~mm}$ in thickness. The root apices were sealed with three layers of nail polish and modeling wax. The specimens were then immersed in $2 \%$ methylene blue for $24 \mathrm{~h}$ at $37^{\circ} \mathrm{C}$. After staining, the teeth were washed with tap water and the nail polish and modeling wax were removed with a scalpel. The teeth were sectioned longitudinally, from buccal to lingual, through the center of the restorations, using a low-speed diamond saw under water-cooling.

\section{Scoring microleakage}

The microleakage was assessed by two calibrated examiners, who were blind to the treatment groups, using a stereomicroscope (SteREO Discovery.V12; Zeiss, Oberkochen, Germany) at $50 \times$ magnification. Two sections were assessed from each tooth near the longitudinal midline of the restoration. Each examiner independently judged the depth of the stain according to standard ranking as shown below. Independent consensus was obtained for each sample from each of the examiners.

Microleakage scores: ${ }^{[8]}$

$0=$ No leakage

$1=$ Dye penetration occurred up to half of the cavity wall depth 
2 =Dye penetration greater than half of the cavity wall depth

$3=$ Dye penetration involving the base of the cavity.

The occlusal wall and the gingival wall were scored separately.

\section{Statistical analysis}

The Kruskal-Wallis test was used to assess differences in microleakage among the different groups. Pair-wise comparisons between groups were performed using the Mann-Whitney test. Statistical significance was defined as $P<0.05$.

\section{RESULTS}

Representative photographic images of microleakage made under a stereomicroscope $(\times 50)$ for each of the six groups are shown in Figure 1a-f. Microleakage scores for groups 1-6 are shown in Table 1.

The results of the Kruskal-Wallis test demonstrated a statistically significant difference in microleakage among groups $(P<0.01)$. Differences were observed for both the occlusal as well as the gingival wall [Table 1].

Bleaching with $10 \%$ carbamide peroxide (groups 2-6) significantly increased microleakage values of restorations compared to the unbleached group (group 1). The unbleached group 1 displayed the least amount of microleakage, while the group in which the filling was done immediately after bleaching (group 2) showed the greatest amount of microleakage [Table 1 and Figure 2].
The delayed filling group 3 and the sodium ascorbate treated group 4 also had a large amount of microleakage that was similar to that in group 2 where the filling was done immediately after bleaching. There was no significant difference between the delayed filling and sodium ascorbate treated groups $(P>0.05)$.

Microleakage in group 5 treated with sodium ascorbate combined with surfactant, and also in the catalase-treated group 6, was significantly $(P<0.05)$ lower compared to group 2 in which the filling was done immediately after bleaching. However, microleakage in these two groups was still significantly greater $(P<0.05)$ than in the unbleached group.

\section{DISCUSSION}

A large number of studies on the interactions between bleaching agents and the bond strength of composite resins to enamel have shown that there is a significant decrease in the bond strength when the composite resin is bonded to bleached enamel compared with unbleached enamel..$^{[-7,13,14,16-18]}$ However, to date, there have been relatively few studies on the increase in the microleakage of composite resin restorations after tooth bleaching, ${ }^{[15]}$ especially external tooth bleaching. Therefore, the aim of this study was to investigative the effects of three antioxidants on the microleakage of composite resin restorations after external tooth bleaching with $10 \%$ carbamide peroxide. Our results showed that bleaching with $10 \%$ carbamide peroxide (groups 2-6) significantly increased the microleakage values of restorations compared to the unbleached group (group 1). This

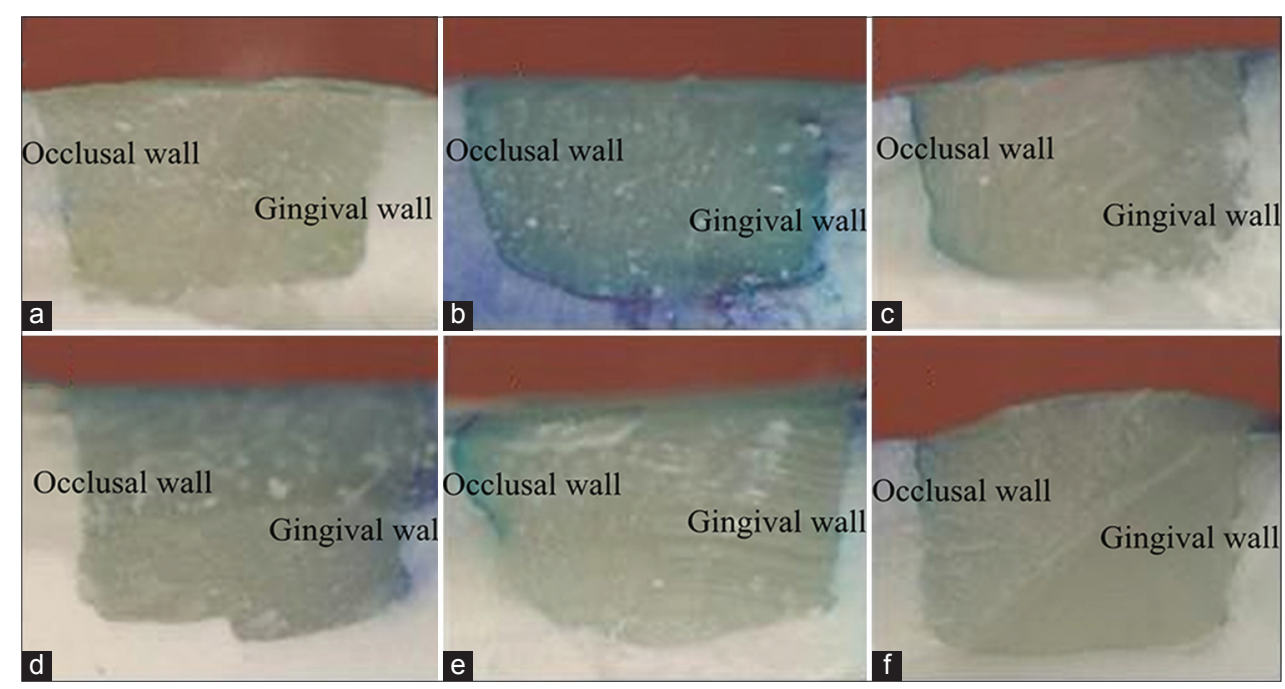

Figure 1: Photographs of six groups under a stereomicroscope (×50): (a) unbleached group (group 1); (b) group in which the filling was done immediately after bleaching (group 2); (c) delayed filling group (group 3); (d) sodium ascorbate treated group (group 4); (e) sodium ascorbate combined with surfactant treated group (group 5); (f) catalase-treated group (group 6) 


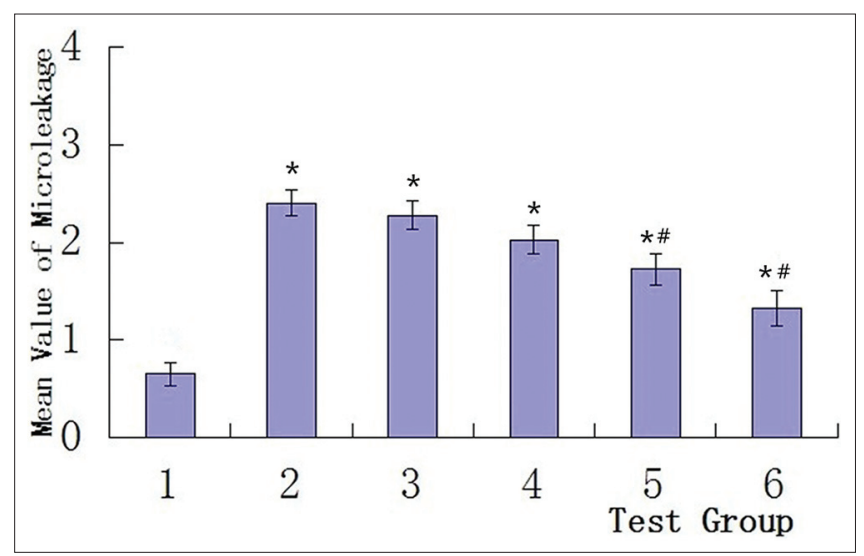

Figure 2: Mean values of microleakage in the test groups. Group 1 displayed the least amount of microleakage, and showed significant differences with groups 2-6 $\left({ }^{*} P<0.01\right)$, Group 2 showed the greatest amount of microleakage, groups 3 and 4 behaved similar to group 2 (not significantly different), while the microleakage in groups 5 and 6 decreased significantly $\left({ }^{\#} P<0.05\right)$ compared to group $2 *: P<0.01$ compared to group 1 , \#: $P<0.05$ compared to group 2

\begin{tabular}{|c|c|c|c|c|c|c|c|c|c|}
\hline \multirow[t]{2}{*}{ Group } & \multirow[t]{2}{*}{ Sample (n) } & \multicolumn{4}{|c|}{ Occlusal wall } & \multicolumn{4}{|c|}{ Gingival wall } \\
\hline & & 0 & 1 & 2 & 3 & 0 & 1 & 2 & 3 \\
\hline 1 & 20 & 4 & 9 & 7 & 0 & 17 & 3 & 0 & 0 \\
\hline 2 & 20 & 0 & 0 & 6 & 14 & 2 & 3 & 6 & 9 \\
\hline 3 & 20 & 0 & 1 & 8 & 11 & 3 & 3 & 4 & 10 \\
\hline 4 & 20 & 0 & 1 & 10 & 9 & 4 & 3 & 9 & 4 \\
\hline 5 & 20 & 0 & 6 & 5 & 9 & 5 & 6 & 7 & 2 \\
\hline 6 & 20 & 0 & 7 & 4 & 9 & 11 & 7 & 2 & 0 \\
\hline
\end{tabular}

may be due to the presence of residual peroxide and oxygen, which has been shown to interfere with resin attachment and inhibit resin polymerization. ${ }^{[6-8]}$ The appearance of the hybrid layer in bleached enamel is less regular and distinct than that in unbleached enamel, and these enamel irregularities may also play a role in the increased microleakage. ${ }^{[21,22]}$

Bulut et al. reported that the residual oxygen left after bleaching may be removed from the enamel surface during the immersion process. ${ }^{[17]}$ In a subsequent study, ${ }^{[23]}$ they showed that immersion of specimens in artificial saliva for 2 weeks could improve the bond strength of composite resin to enamel after bleaching. This finding was not supported by the results of our current study, where the delayed filling group accumulated microleakage that was not significantly different from microleakage in the immediate restoration group.

In this study, we found significant differences when comparing microleakage in the bleached control group to that of groups that had antioxidant treatments (groups 5 and 6). As in previous studies, ${ }^{[-8-8,12,16,17]}$ these results suggest that antioxidants play an important role in decreasing microleakage when restorations are done immediately after bleaching. Moreover, in our study, except for the unbleached group, the lowest microleakage values were observed in samples treated with new antioxidants, such as sodium ascorbate combined with Tween 80 (group 5), or with catalase (group 6). Ascorbic acid and its salts are well-known antioxidants that can reduce a variety of oxidative compounds, especially free radicals. ${ }^{[15,17,24]}$ Moreover, ascorbic acid (Vitamin C) and its salts are non-toxic and have been widely used in the food industry. Thus, they have no adverse biological effects or clinical hazards ${ }^{[15]}$ when used in the oral cavity. Lai et al. ${ }^{[25]}$ suggested that sodium ascorbate allowed free radical polymerization of the adhesive to proceed without premature termination by restoring the altered redox potential of the oxidized bonding substrate and eventually reversing the compromised bonding.

Sodium ascorbate was used in solution form in the present study and in previous studies. However, sodium ascorbate solution is more difficult for practitioners and patients to manipulate than the hydrogel form. The hydrogel form of sodium ascorbate can be placed in the bleaching tray before bonding. This is less expensive due to the shorter chair time needed. Some studies have shown that there is no significant difference in microleakage between the two forms of sodium ascorbate (hydrogel and solution), ${ }^{[14]}$ but other studies have reported that the hydrogel form is better. ${ }^{[26]}$ There have been few studies researching sodium ascorbate hydrogel, and it could be a novel research area for future development of a factory-made antioxidant.

Notably, there were no significant differences found in microleakage between the group treated with only sodium ascorbate (group 4) and the delayed filling group (group 3). One reason may be that the application time of the antioxidants was too short. In a study carried out by Bulut et al., ${ }^{[17]}$ sodium ascorbate solution was used for $10 \mathrm{~min}$ in order to neutralize the oxidizing effect of carbamide peroxide. Meanwhile, Lai et al..$^{[15]}$ used sodium ascorbate solution for $3 \mathrm{~h}$ and suggested that sodium ascorbate should be used for at least one-third of the amount of time that the oxidizing bleaching agent was applied (e.g. bleaching gel was generally applied for $8 \mathrm{~h}$ a day). However, it is time consuming and impractical to apply the antioxidants for this proposed length of time prior to composite resin restoration. As an example, the sodium ascorbate hydrogel would need to be placed in the bleaching tray for $3 \mathrm{~h}$ by the patients themselves before bonding. 
In the present study, the antioxidants were used in group 4 for only $1 \mathrm{~min}$ in order to correspond with the catalase group. As with a previous study, the sodium ascorbate, by itself, had no significant effect on microleakage. Thus, in future studies, the application time will be extended to $10 \mathrm{~min}$.

Moosavi et al. ${ }^{[5]}$ proposed that a bleached surface in the access cavity may reduce the surface tension and contact angle, as well as the cohesive force between the sodium ascorbate molecules after treatment with $10 \%$ sodium ascorbate gel in combination with $0.2 \%$ Tween 80. There may be two reasons for this. First, $0.2 \%$ Tween 80 is a nonionic surfactant that can allow the antioxidant agent to easily penetrate the dentin, improving the efficacy in the dentinal tubules and enamel. ${ }^{[5]}$ Second, $0.2 \%$ Tween 80 's detergent effect on the surface can improve the sealing of composite resin by increasing the free surface energy of the tooth. ${ }^{[5]}$ Accordingly, the 3-h length of time for antioxidant treatment could be decreased when the antioxidant is combined with this surfactant, and this was supported by our study. Our results showed that when sodium ascorbate combined with $0.2 \%$ Tween 80 was used for 1 min on the cavity, microleakage decreased significantly.

Rotstein et al ${ }^{[19]}$ reported that catalase can effectively eliminate residual hydrogen peroxide when used as an adjunct following intracoronal bleaching of nonvital teeth. Kum et al. ${ }^{[18]}$ also found that pretreatment of a bleached surface with catalase prior to bonding significantly improved the composite-enamel bond strength. Similarly, our results suggested that catalase should be recommended for clinical use since it significantly reversed the adverse effects of peroxide and oxygen on microleakage associated with composite resin restorations after external tooth bleaching, and it was effective after a short application time. However, further studies need to be done to optimize the efficacy of catalase for this purpose.

Studies on the effects of other antioxidant agents for neutralizing the adverse effects of bleaching materials on composite resin restorations have not been done. Two other antioxidants, 10\% ascorbic acid and VitE, have displayed high antioxidant activity among the substances tested by DPPH (1,1-diphenyl-2-picrylhydrazine) guided fractionation assay. ${ }^{[27]}$ However, $10 \%$ ascorbic acid is inappropriate for clinical use due to its very low $\mathrm{pH}$ value (about 1.8). It is difficult to store it for a long period of time because the solution gradually oxidizes over time during storage to become less reductive. VitE, a lipid-soluble antioxidant ( $\mathrm{pH}$ 6.8), has recently been shown to have good antioxidant activity in dentin and enamel, similar to ascorbic acid. ${ }^{[13]}$ This may be due to the presence of alcohol in the composition of the VitE, as previous studies have reported that applying alcohol to the bleached enamel increases the bond strength, although not to the level of the non-bleached teeth. ${ }^{[17]}$ This hypothesis needs to be tested further.

The depth of dye penetration at the interface between the wall of the cavities and the restorations is adopted as a criterion by most studies on microleakage, but ordinally ranked observational data is only semi-quantitative and incorporates subjective variability. Further studies are needed in which the depth of the dye penetration in the cavity is measured directly on all specimens using a stereomicroscope, thus providing continuous data and more precision.

In studying microleakage caused by bleaching agents, the scanning electron microscope (SEM) may be useful to observe the ultrastructure of specimens with microleakage. Several authors have used SEM and reported that increased porosity of enamel is manifested by an over-etched appearance with loss of prismatic structure. ${ }^{[28]}$ In the study of Türkün and Kaya, the interface between resin and bleached enamel, observed on SEM, displayed a granular and porous aspect with a bubbly appearance that may have been the result of oxidized peroxide that was trapped in the subsurface layer of the enamel. ${ }^{[7]}$

In the future, teeth that have been treated with our experimental antioxidant formulation should be assessed for their microstructure and bond strength characteristics. Further, it should be determined whether the higher microleakage rates that were observed in vitro subsequent to bleaching translate to significant deterioration of restorations in actual clinical situations and whether this deterioration can be reduced in the clinic using an effective antioxidant formulation. In addition, determining the minimum time required for antioxidant treatment after external tooth bleaching will be necessary. Therefore, further studies are needed to better understand these issues and address practical concerns in terms of clinical applications.

\section{CONCLUSIONS}

Microleakage increased significantly after external bleaching with $10 \%$ carbamide peroxide, and 
decreased when the bleached teeth were treated with sodium ascorbate combined with Tween 80, or with catalase. Catalase was more effective at decreasing microleakage, while delayed filling or treatment with sodium ascorbate alone did not effectively decrease the microleakage of composite resin restorations.

\section{ACKNOWLEDGMENTS}

This work was supported by the National Natural Science Foundation of China (Grant Nos 81300867, 81271134), Doctoral Innovation Fund of Shanghai Jiao Tong University (Grant No. BXJ201225), and Specialized Research Fund for the Doctoral Program of Higher Education (Grant No. 20130073110013). The authors deny any conflicts of interest related to this study.

\section{REFERENCES}

1. Matis BA, Cochran MA, Eckert G, Carlson TJ. The efficacy and safety of a 10\% carbamide peroxide bleaching gel. Quintessence Int 1998; 29:555-63.

2. Leonard RH, Sharma A, Haywood VB. Use of different concentrations of carbamide peroxide for bleaching teeth: An in vitro study. Quintessence Int 1998;29:503-7.

3. Türkün M, Türkün LS. Effect of nonvital bleaching with $10 \%$ carbamide peroxide on sealing ability of resin composite restorations. Int Endod J 2004;37:52-60.

4. Ulukapi H, Benderli Y, Ulukapi I. Effect of pre- and postoperative bleaching on marginal leakage of amalgam and composite restorations. Quintessence Int 2003;34:505-8.

5. Moosavi H, Moghaddas MJ, Ghoddusi J, Rajabi O. Effects of two antioxidants on the microleakage of resin-based composite restorations after nonvital bleaching. J Contemp Dent Pract 2010;11:E033-40.

6. Attin T, Hannig C, Wiegand A, Attin R. Effect of bleaching on restorative materials and restorations-a systematic review. Dent Mater 2004;20:852-61.

7. Türkün M, Kaya AD. Effect of $10 \%$ sodium ascorbate on the shear bond strength of composite resin to bleached bovine enamel. J Oral Rehabil 2004;31:1184-91.

8. Kaya AD, Türkün M, Arici M. Reversal of compromised bonding in bleached enamel using antioxidant gel. Oper Dent 2008;33:441-7.

9. Bittencourt ME, Trentin MS, Linden MS, de Oliveira Lima Arsati YB, França FM, Flório FM, et al. Influence of in situ postbleaching times on shear bond strength of resin-based composite restorations. J Am Dent Assoc 2010;141:300-6.

10. Da Silva Machado J, Cândido MS, Sundfeld RH, De Alexandre RS, Cardoso JD, Sundefeld ML. The influence of time interval between bleaching and enamel bonding. J Esthet Restor Dent 2007;19:111-9.

11. Kaya AD, Türkün M. Reversal of dentin bonding to bleached teeth. Oper Dent 2003;28:825-9.

12. Muraguchi K, Shigenobu S, Suzuki S, Tanaka T. Improvement of bonding to bleached bovine tooth surfaces by ascorbic acid treatment. Dent Mater J 2007;26:875-81.
13. Sasaki RT, Flório FM, Basting RT. Effect of $10 \%$ sodium ascorbate and $10 \%$ alpha-tocopherol in different formulations on the shear bond strength of enamel and dentin submitted to a home-use bleaching treatment. Oper Dent 2009;34:746-52.

14. Torres CR, Koga AF, Borges AB. The effects of anti-oxidant agents as neutralizers of bleaching agents on enamel bond strength. Braz J Oral Sci 2006;5:971-6.

15. Lai SC, Tay FR, Cheung GS, Mak YF, Carvalho RM, Wei SH, et al. Reversal of compromised bonding in bleached enamel. J Dent Res 2002;81:477-81.

16. Kimyai S, Oskoee SS, Rafighi A, Valizadeh H, Ajami AA, Helali ZN. Comparison of the effect of hydrogel and solution forms of sodium ascorbate on orthodontic bracket-enamel shear bond strength immediately after bleaching: An in vitro study. Indian J Dent Res 2010;21:54-8.

17. Bulut H, Turkun M, Kaya AD. Effect of an antioxidizing agent on the shear bond strength of brackets bonded to bleached human enamel. Am J Orthod Dentofacial Orthop 2006;129:266-72.

18. Kum KY, Lim KR, Lee CY, Park KH, Safavi KE, Fouad AF, et al. Effects of removing residual peroxide and other oxygen radicals on the shear bond strength and failure modes at resin-tooth interface after tooth bleaching. Am J Dent 2004;17:267-70.

19. Rotstein I. Role of catalase in the elimination of residual hydrogen peroxide following tooth bleaching. J Endod 1993;19:567-9.

20. Cavalli V, Reis AF, Giannini M, Ambrosano GM. The effect of elapsed time following bleaching on enamel bond strength of resin composite. Oper Dent 2001;26:597-602.

21. Titley KC, Torneck CD, Smith DC, Chernecky R, Adibfar A. Scanning electron microscopy observations on the penetration and structure of resin tags in bleached and unbleached bovine enamel. J Endod 1991;17:72-5

22. Barkhordar RA, Kempler D, Plesh O. Effect of nonvital tooth bleaching on microleakage of resin composite restorations. Quintessence Int 1997;28:341-4.

23. Kunt GE, Yilmaz N, Sen S, Dede DÖ. Effect of antioxidant treatment on the shear bond strength of composite resin to bleached enamel. Acta Odontol Scand 2011;69:287-91.

24. Bulut H, Kaya AD, Turkun M. Tensile bond strength of brackets after antioxidant treatment on bleached teeth. Eur J Orthod 2005;27:466-71.

25. Lai SC, Mak YF, Cheung GS, Osorio R, Toledano M, Carvalho RM, et al. Reversal of compromised bonding to oxidized etched dentin. J Dent Res 2001;80:1919-24.

26. Dabas D, Patil AC, Uppin VM. Evaluation of the effect of concentration and duration of application of sodium ascorbate hydrogel on the bond strength of composite resin to bleached enamel. J Conserv Dent 2011;14:356-60.

27. Garcia EJ, Oldoni TL, Alencar SM, Reis A, Loguercio AD, Grande RH. Antioxidant activity by DPPH assay of potential solutions to be applied on bleached teeth. Braz Dent J 2012;23:22-7.

28. Josey AL, Meyers IA, Romaniuk K, Symons AL. The effect of a vital bleaching technique on enamel surface morphology and the bonding of composite resin to enamel. J Oral Rehabil 1996;23:244-50.

\begin{tabular}{|l|l|}
\hline \multicolumn{2}{|c|}{ Access this article online } \\
\hline Quick Response Code: & Website: \\
& www.eurjdent.com \\
\cline { 2 - 2 } & \\
\hline
\end{tabular}

